\title{
A one-day, 90-minute aspirin challenge and desensitization protocol in aspirin-exacerbated respiratory disease
}

\author{
Geneva A. DeGregorio, M.D. ${ }^{1}$, Joseph Singer, B.A. ${ }^{2}$, Katherine N. Cahill, M.D. ${ }^{3}$, and Tanya \\ Laidlaw, M.D. ${ }^{2}$ \\ ${ }^{1}$ Department of Medicine, Brigham and Women's Hospital, Boston, Massachusetts \\ 2Department of Medicine, Harvard Medical School, the Division of Rheumatology, Immunology \\ and Allergy, Brigham and Women's Hospital, and the Jeff and Penny Vinik Center, Boston, \\ Massachusetts. \\ ${ }^{3}$ Divison of Allergy, Pulmonary and Critical Care Medicine, Department of Medicine, Vanderbilt \\ University, Nashville, Tennessee
}

\begin{abstract}
Background: Aspirin challenge and desensitization remains the gold standard in diagnosis and treatment for patients with aspirin-exacerbated respiratory disease (AERD), but the protocols can be time and resource intensive.
\end{abstract}

Objective: To provide evidence that oral aspirin challenge and desensitization can be safely performed in an outpatient setting in one day.

Methods: Forty-four patients with a confirmed diagnosis of AERD, stable asthma and baseline FEV1 $\geq 70 \%$ of predicted, completed an oral aspirin challenge and desensitization protocol. The starting dose was $40.5 \mathrm{mg}$ with escalating doses of aspirin $(81,162.5,325 \mathrm{mg})$ at 90 -minute intervals until symptoms were provoked. Desensitization was defined as tolerating a repeated administration of the provocative aspirin dose and at least one subsequent dose, bringing the total aspirin ingested during the in-clinic desensitization to $2325 \mathrm{mg}$.

Results: Ninety-three percent of patients completed the challenge and desensitization in one day, with an average protocol completion time of 9 hours and 29 minutes. Two patients (4.6\%) chose to complete the protocol over two days. One patient (2.3\%) was discontinued from the protocol due to on-going abdominal discomfort and diarrhea. No patient required epinephrine, emergency department visit or hospitalization.

Corresponding Author: Tanya M. Laidlaw, MD, Address: Brigham and Women's Hospital, 60 Fenwood Road, Building of Transformative, Medicine, Rm 5002M, Boston, MA 02115, Phone: 617-525-1034, Fax: 617-525-1310, tlaidlaw@bwh.harvard.edu. Conflict of Interest: T Laidlaw has served on scientific advisory boards for GlaxoSmithKline and Sanofi-Genzyme. K Cahill has served on scientific advisory boards for Teva, Optinose, and Regeneron. GA DeGregorio and J Singer and have no conflicts of interest to disclose.

Publisher's Disclaimer: This is a PDF file of an unedited manuscript that has been accepted for publication. As a service to our customers we are providing this early version of the manuscript. The manuscript will undergo copyediting, typesetting, and review of the resulting proof before it is published in its final citable form. Please note that during the production process errors may be discovered which could affect the content, and all legal disclaimers that apply to the journal pertain. 
Conclusions: Patients with AERD on a stable asthma regimen and with a baseline FEV1 $\ 70 \%$ can be safely desensitized to aspirin using a 90-minute dose escalation protocol, starting at a dose of $40.5 \mathrm{mg}$, and defining desensitization as tolerance of the repeated provocation dose and at least one subsequent aspirin dose, bringing total cumulative daily dose to $325 \mathrm{mg}$. This protocol can routinely be completed in one day.

\section{Keywords}

Aspirin-exacerbated respiratory disease; AERD; NSAID-exacerbated respiratory disease; NERD; aspirin challenge; aspirin desensitization

\section{INTRODUCTION}

Aspirin-exacerbated respiratory disease (AERD) refers to the triad of asthma, chronic rhinosinusitis with nasal polyposis and acute upper and lower respiratory tract reactions following ingestion of aspirin or other non-steroidal anti-inflammatory drugs (NSAIDs) that inhibit COX-1. Although NSAID avoidance is recommended for AERD, patients who require aspirin therapy for refractory nasal polyposis, difficult to control asthma, or inflammatory or cardiovascular disease can undergo aspirin challenge and desensitization. ${ }^{1}$

Aspirin challenge and desensitization requires delivering escalating doses of aspirin or another COX-1 inhibitor to patients with AERD, provoking a reaction, persisting to establish tolerance and then continuing long-term therapy without interruption. ${ }^{2}$ Patients are then able to benefit from the therapeutic effects of high-dose aspirin. Traditionally, aspirin desensitization took place over the course of three or more days using a 3-hour escalation protocol. $^{1,2}$ Beginning with a 30mg dose at 8:00am on Day 1, followed three hours later by $60 \mathrm{mg}$, and continued escalation every three hours to 100, 150, 325 and 650mg. Symptomprovoking doses were repeated until the patient demonstrated tolerance. Although the lengthy protocol was designed to prevent severe bronchospastic and anaphylactic reactions requiring aggressive treatment or hospitalization, it was time and resource intensive for practitioner, patient and health system.

There are now a variety of aspirin challenge and desensitization protocols that differ in dose escalation time (60-minute, 90-minute, 3-hour), starting dose $(20.25 \mathrm{mg}, 40.5 \mathrm{mg})$, and route of COX-1 inhibitor administration (oral aspirin vs intranasal ketorolac vs intranasal lysine acetylsalcylate) (Table 1). ${ }^{1,3-9}$ After a study showed that reactions are usually provoked at or before the $325 \mathrm{mg}$ dose, modern challenge protocols now end with a maximum dose of $325 \mathrm{mg} .{ }^{10}$ Many studies report that patients rarely react to low doses such as 20 or $30 \mathrm{mg}$, suggesting that it may be safe in most circumstances to begin with a $40.5 \mathrm{mg}$ dose, though this has not yet become standard practice. ${ }^{11}$

Although oral aspirin protocols are the simplest logistically and least time and resource intensive, intranasal ketorolac protocols have demonstrated excellent safety profiles including increasing the percentage of patients with naso-ocular reactions only. ${ }^{6}$ Intranasal lysine acetylsalicylate is not available in the United State of America, but is available in Japan and most of Europe and has a similar safety profile when used for intranasal challenges. ${ }^{8}$ 
There are two published protocols with an escalation time of 60-minutes. The first begins at $40.5 \mathrm{mg}$ and increases to $81,120,162$ and $325 \mathrm{mg}$, but the study excluded $15 \%$ of patients due to a history of reaction time greater than 1 hour. ${ }^{5}$ Mean time to reaction was 50-minutes with a standard deviation of 21-minutes suggesting that the 60-minute escalation time in some cases allows for stacking of escalating doses and may put patient safety at risk. The second begins with $40 \mathrm{mg}$ and increases to 80,160 and $325 \mathrm{mg}$ and delivers aspirin in an Alka-Seltzer® solution. ${ }^{9}$ This study reports no difference in safety compared to a 90 -minute escalation protocol. The Aspirin Desensitization Joint Task Force recommended a 90-minute escalation protocol starting at $20.25 \mathrm{mg}$ and increasing to $40.5,81,162.5$ and $325 \mathrm{mg}$, but unfortunately there are limited data available regarding the safety or efficacy of this approach. ${ }^{3,4}$

The aim of this paper is to document the safety and tolerability of a one-day protocol for oral aspirin challenge and desensitization in AERD patients, performed in the outpatient setting at the Brigham and Women's Hospital AERD Center. Specifically, we describe the implementation of a 90-minute dose escalation and 40.5mg starting dose. Additionally, we propose that patients can be considered fully desensitized after tolerating a repeated administration of the provocative dose and at least one subsequent aspirin dose, bringing the total aspirin ingested during the in-clinic desensitization to $2325 \mathrm{mg}$. Although historically we had required that patients remain in the clinic until they had demonstrated that they could tolerate a full single dose of $325 \mathrm{mg}$ of aspirin, we now have evidence to justify our current practice, which allows most patients to go home prior to ingesting a full $325 \mathrm{mg}$ dose. These elements decrease protocol length and increase likelihood of completion within one day.

\section{METHODS}

\section{Participants and Inclusion and Exclusion Criteria}

Patients with AERD participated in one of two institutional review board approved clinical studies: "Role of inflammatory mediators in aspirin-exacerbated respiratory disease" [NCT02824523] or "Therapeutic Control of Aspirin-Exacerbated Respiratory disease" [NCT01597375] between August 2012 and June 2018 at Brigham and Women's Hospital. ${ }^{11}$ Forty-four patients met protocol-defined criteria for AERD, were eligible for aspirin challenge and desensitization and their data were included in this study.

Inclusion criteria comprised patients aged 18 to 75 , documented history of asthma, nasal polyps, chronic rhinosinusitis, and at least one clinical adverse reaction to any COX-1 inhibitor, stable asthma (baseline $\mathrm{FEV}_{1} \geq 70 \%$ of predicted and no hospitalization or emergency room visits for asthma for at least the prior six months), stable asthma treatment for a minimum of four weeks, and oral montelukast $10 \mathrm{mg}$ daily for a minimum of two weeks prior to aspirin challenge. Exclusion criteria included severe gastro-esophageal reflux, history of peptic ulcer disease, gastrointestinal bleeding, current use of anticoagulant or antiplatelet drugs, pregnant or breast feeding, current smoker, or use of oral beta-blockers. Patients continued regular asthma controller medications including long acting beta agonists, intranasal and inhaled corticosteroids, stable doses of oral glucocorticoids, and could continue antihistamines if used regularly. Patients taking zileuton at time of screening underwent a four-week washout period prior to challenge. 


\section{Materials}

Patients were administered oral aspirin in escalating doses (40.5, 81, 162.5, and 325mg), based on commercially-available pill sizes of $81 \mathrm{mg}$ and $325 \mathrm{mg}$ with the intermediate doses achieved with a pill cutter. During the three-hour period of observation following the onset of reaction symptoms, the only rescue medications permitted were nebulized albuterol sulfate and ipratropium for decreased lung function, and epinephrine for anaphylaxis. Following that three-hour period of observation, remaining reaction symptoms could be treated with oral ondansetron for nausea, zileuton for gastrointestinal pain or skin rashes, or prednisone for severe respiratory reactions, depending on patient comfort.

\section{Procedure for Oral Aspirin Challenge and Desensitization}

Prior to aspirin administration, a physical exam and spirometry were performed. Additionally, results from the total nasal symptoms score (TNSS) and Asthma Control Questionnaire (ACQ) were recorded at baseline. The TNSS questionnaire is a 9-symptom patient recorded outcome measurement on a total scale of 0-40, which includes assessment of nasal congestion, runny nose, sneezing, nasal itching, and eye and throat symptoms. ${ }^{11}$

Challenge began with administration of $40.5 \mathrm{mg}$ oral aspirin followed by a 90 -minute interval, and then repeat spirometry, vital sign measurements, and TNSS administration. If no reaction occurred after 90 minutes, the procedure was repeated with sequentially increasing doses of oral aspirin $(81,162.5$, and $325 \mathrm{mg}$ ) every 90 minutes until a reaction was elicited. Once the onset of reaction occurred, as defined by either a decrease in forced expiratory volume in 1 second (FEV1) of $\geq 15 \%$ from baseline or a worsening of least 2 of the symptoms as reported on the TNSS, the patient was observed for three hours without further administration of aspirin.

Following the three-hour observation period, FEV1 and TNSS were measured. Patients demonstrating FEV1 within $85 \%$ of baseline and TNSS downtrend proceeded to repeat administration of provocative dose. If these criteria were not met, patients were observed or treated with nebulized albuterol until they met criteria. After repeat provocative dose, the patient was again observed for 90-minutes and spirometry, vital sign measurements, and TNSS administration was repeated. If the repeated provocative dose elicited onset of a further reaction (as defined by criteria listed previously), the patient waited additional 90 minutes and then the provocative dose was administered a third time. If the repeated provocative dose was tolerated, the next highest dose was administered. This process was repeated until the patient had received a total of at least $325 \mathrm{mg}$ aspirin that day and had received both the repeated provocative dose and the next sequential dose without further reaction. There were 29 patients eligible to complete desensitization without having taken the full $325 \mathrm{mg}$ dose, but 15 patients, either due to patient preference or earlier protocol version requirement, remained in the clinic to receive it. Following the final dose administered, the patient was observed for 90 minutes and spirometry, vital sign measurements, and TNSS were repeated. The patient was then given discharge instructions to begin $325 \mathrm{mg}$ aspirin twice a day at home the following day, and to continue for one week. After one week they were instructed to increase to $650 \mathrm{mg}$ twice a day. Patients were instructed to call the clinic if they experienced any further upper or lower respiratory, 
gastrointestinal, dermatologic or other concerning symptoms and they were evaluated in in clinic after eight weeks on aspirin 650mg twice daily.

\section{Data Analysis}

All study data were collected on standardized case report forms and the data were entered in REDCap. Data were exported and descriptive statistics were performed in Microsoft Excel. Means and standard deviations are reported unless otherwise specified.

\section{RESULTS}

\section{Patient characteristics}

Forty-four patients underwent the aspirin challenge and desensitization protocol. Most patients were on both inhaled corticosteroids and a long acting inhaled bronchodilator for daily asthma control, their asthma was well-controlled based on ACQ score, and baseline nasal symptoms were generally minimal as recorded by TNSS on the morning of their aspirin desensitization (Table 2). All patients took montelukast 10mg daily for at least two weeks prior to aspirin administration.

\section{Provocative aspirin dose and time}

The provocative dose was $40.5 \mathrm{mg}$ for $9.1 \%$ of patients, $81 \mathrm{mg}$ for $56.7 \%, 162.5 \mathrm{mg}$ for $29.6 \%$ and $325 \mathrm{mg}$ for $2.3 \%$. There was one patient $(2.3 \%)$ with prior proven AERD who did not react and was considered to have a "silent desensitization". Based on our dosing schedule, this meant that two-thirds (66\%) of patients reacted to 40.5 or $81 \mathrm{mg}$ and thus had their onset of reaction before 11:00am (Figure 1).

\section{Symptomatic reaction to provocative dose}

The average time to reaction following dose administration was 61 minutes (SD 22 minutes), the minimum time to reaction was 19 minutes and the maximum time to reaction was 99 minutes (Table 3). Two patients had a reaction time greater than the allotted 90 minute observation period (92 and 99 minutes). In both cases, the patient was having mild subjective symptoms that suggested the start of a provoked reaction but did not yet meet study criteria for provocation as outlined above. For the purpose of safety, they were observed for an additional few minutes and TNSS and FEV1 were repeated at which time the patients met defined criteria for the onset of reaction. All patients developed naso-ocular symptoms, with 50\% developing no lower respiratory reaction, and $47.7 \%$ experiencing additional symptoms of a bronchial reaction, defined as a decrease in FEV1 of $\geq 15 \%$ from baseline. No patients experienced systemic hypotension. Just under $20 \%$ of patients experienced dermatologic reactions (skin flushing and/or rash) and just over 20\% experienced gastrointestinal symptoms (nausea, vomiting, or diarrhea).

Patients were observed for a minimum of three hours following onset of upper or lower respiratory tract symptoms. On average, the FEV1 nadir occurred at one hour after onset of reaction (Figure 2A). Of the patients whose FEV1 did fall notably during their reaction, the median FEV1 nadir was $-26 \%$ of predicted. The largest decrease in FEV1 was $-66 \%$ of predicted. On average, peak nasal symptoms occurred at 1.5 hours (Figure 2B). Following 
the three-hour observation period, 35 patients were able to immediately repeat their provocative dose as their FEV1 was within $85 \%$ of baseline and their TNSS had peaked and was downtrending. Five patients still had a decline in FEV1 of $\geq 15 \%$ and required further treatments with nebulized albuterol. These five patients were all able to repeat their provocative dose within 4.5 hours from the onset of reaction.

\section{Feasibility of one-day protocol}

In our study, 41 of $44(93 \%)$ patients were able to complete the aspirin challenge and desensitization protocol in one day. Of the three patients (6.8\%) who did not complete the desensitization in one day, one had significant abdominal discomfort and diarrhea such that the supervising physician discontinued the protocol and one patient chose to come back and complete the following day for their own convenience. A third patient's FEV1 did not return to within $85 \%$ of baseline until 4.5 hours after the onset of the reaction, so the repeated administration of the provocative dose did not occur until 4:00pm. Therefore, due to time constraints, this patient chose to come back and complete the following day. Among those who completed challenge and desensitization in one day, the average length of time in clinic was 9 hours and 29 minutes.

The study defined desensitization as tolerating a repeat administration of the provocative dose and at least one subsequent aspirin dose, provided that the total aspirin ingested during the day was at least $325 \mathrm{mg}$. As such there were 29 patients who were eligible to complete desensitization without having taken a full $325 \mathrm{mg}$ dose in the clinic. Despite that, there were 15 patients who stayed to complete the entire dose-escalation process including the $325 \mathrm{mg}$ dose. None of these patients developed symptoms of further reaction following the $325 \mathrm{mg}$ dose.

All patients who achieved desensitization as above began taking $325 \mathrm{mg}$ aspirin twice daily on the morning after the desensitization procedure and none of those patients developed any further symptoms of reaction upon ingestion of the next morning's 325mg dose.

\section{DISCUSSION}

The gold standard for treatment of AERD is oral aspirin challenge and desensitization to initiate high-dose aspirin therapy. Historically, this has been a time and resource intensive process that occurred over the course of two or more days. There are now several protocols for aspirin challenge and desensitization that vary based on dose escalation time, starting dose and route of COX-1 inhibitor administration with the aim of improving safety and efficiency of this process. Here we document a 90-minute escalation protocol for oral aspirin challenge and desensitization that can be routinely completed in one day, decreasing time and resource demand on patient, practitioner and health system while preserving patient safety.

\section{Protocol design and comparison}

An optimal aspirin challenge and desensitization protocol would maintain an exceptional safety profile while minimizing time and resource utilization. Possible ways to decrease protocol length include decreasing the length of time between each dose escalation, 
beginning the protocol at a higher starting dose and liberalizing the requirements to define achievement of desensitization. Our study implements all these approaches and 93\% of patients safely completed the protocol in one day.

The length of time between dose escalation has varied by protocol. The original protocol for aspirin desensitization used a 3 -hour dose escalation. ${ }^{1}$ This has proven safe; however, the time burden is high as the protocol usually requires three days of testing. The 90-minute dose escalation protocol is recommended by Aspirin Desensitization Joint Task Force; however this is primarily based on expert opinion as there has been little data documenting safety of 90-minute escalation interval. 3,4 Two studies have examined the use of a 60-minute dose escalation protocol. The first was in patients whose reported usual onset of symptoms was within one hour of exposure to a COX-1 inhibitor. ${ }^{5}$ The average time to reaction was 50.3 minutes with standard deviation of 20 minutes, which raises concern for risk of dose stacking before a reaction has become evident, potentially compromising patient safety. The study also examined seven patients who gave a history of reaction time greater than one hour by history and when these seven patients underwent the three-hour dose-escalation protocol the mean time to symptom onset was 70 minutes. In the second study, the average time to reaction was 46 minutes with a standard deviation of 4 minutes. ${ }^{9}$ This reaction time is one of the shortest in the literature and the standard deviation is extremely narrow. This study administered aspirin in an Alka-Seltzer® solution, which may allow for faster aspirin absorption and may decrease the time to provoked reaction, and it also had more frequent peak expiratory flow, nasal peak flow and spirometry monitoring which may have hastened the reporting and earlier detection of patient symptoms. ${ }^{12}$ In one study of 28 patients with aspirin hypersensitivity (manifest as asthma, rhinosinusitis, urticaria, angioedema and anaphylaxis) there were 21 patients who completed a two-hour dose escalation protocol and seven patients who completed a 1.5-hour dose escalation protocol. They found that all positive reactions occurred within 90 -minutes of administration. ${ }^{13}$ In our study, which included patients regardless of their reported historic reaction time, we found the mean time from provocative dose administration to symptom onset was 62 minutes. Taken together, we conclude that the 90-minute dose escalation protocol is safe and can be routinely used in lieu of a 3-hour escalation. A 60-minute dose escalation protocol, perhaps including administration of diluted aspirin in solution and more frequent objective monitoring for provoked reaction, may have promise, but there is not yet sufficient evidence to adopt this protocol widely.

The starting dose for aspirin challenge and desensitization also varies, with most protocols recommending a conservative dose (20 or $30 \mathrm{mg}$ ) or a range (20-40 mg or $30-60 \mathrm{mg}$ ) to be decided based on risk for severe reaction. A study examining predictors of reaction severity found that among patients taking a leukotriene-modifying drug, those with baseline FEV1 $<80 \%$ of predicted or with history of recent ED visit for asthma had a significantly higher risk of moderate or severe reaction during aspirin challenge and therefore recommended that patients with low or moderate risk of significant bronchial reactions start at 40 to $60 \mathrm{mg}$ and patients with a history of ED visit for asthma or low baseline FEV1 and thus high risk for significant bronchial reactions start at lowest available 20-30mg dose. ${ }^{10}$ Our study, which included patients with FEV1 $270 \%$ of predicted and stable asthma in the weeks prior to challenge/desensitization, but did not exclude based on history of ED visit or hospitalization 
for asthma, started all patients at a $40.5 \mathrm{mg}$ dose. Only four patients (10\%) reacted to this lowest dose and none had severe reactions requiring discontinuation or modification of the protocol. Given that the clear majority of patients do not react to the $40.5 \mathrm{mg}$ starting dose and those who do will tolerate this well, we favor starting at this dose for patients with FEV1 $\geq 70 \%$ and stable asthma.

The threshold at which a patient was considered tolerant to aspirin has also changed over time. In the original protocol, aspirin tolerance was defined as tolerating a dose of $650 \mathrm{mg}$. More recently, after a study showed that reactions always occur at or before the $325 \mathrm{mg}$ dose, aspirin tolerance and a "negative challenge" has been declared following tolerance of a full $325 \mathrm{mg}$ dose.${ }^{10}$ However, declaring a desensitization as successful at a lower threshold than this has never been studied. The standard practice at the Brigham and Women's Hospital AERD Center, based on expert opinion, is to define desensitization as tolerance of repeat provocation dose and one subsequent dose bringing total cumulative daily dose to $2325 \mathrm{mg}$. This means that the large group of patients who react to the $81 \mathrm{mg}$ dose will repeat that dose and then be administered the $162 \mathrm{mg}$ dose but will not have to demonstrate tolerance of the full 325mg dose while in the clinic. This ultimately decreases the length of time to complete the protocol.

An additional consideration in the aspirin challenge and desensitization protocols is that of the route of administration of the COX-1 inhibitor. Although the initial published protocols used only oral aspirin, studies of intranasal ketorolac sprays administered every 30 minutes for four escalating doses and then transition to a three-hour oral aspirin challenge starting at $60 \mathrm{mg}$ were found to be safe and effective alternative for diagnosing AERD and desensitizing to aspirin. ${ }^{6,7}$ These studies found that that in comparison to the three-hour escalation oral aspirin protocol, the intranasal ketorolac and aspirin protocols were shorter (1.9 vs 2.6 days on average) and more patients were able to complete the desensitization by day 2 ( $83 \mathrm{vs}$ $18 \%$ ). The ketorolac and aspirin protocols also induced less decline in FEV1 (mean decrease of $8.5 \mathrm{vs} 13.4 \%$ ), demonstrated a higher percentage of patients who had no lower respiratory symptoms (54 vs $35 \%$ ), and caused fewer extrapulmonary symptoms (gastric symptoms or cutaneous reactions). Although advantageous for the reasons listed above, the ketorolac protocol has a few drawbacks: 1) it cannot be completed in one day, 2) it requires the use of two COX-1 inhibitor products, and 3) the intranasal formulation of ketorolac is not commercially available and has to be mixed specially for this purpose. Furthermore, the false negative rate of the intranasal challenge protocols for the diagnosis of AERD is higher than for the pure oral aspirin challenge protocols, making them less useful from a diagnostic perspective.

Finally, although not implemented in this study, there is some evidence to show that manipulation of pharmacokinetics of aspirin onset of action by asking patients to chew and swallow aspirin or to administer in a solution could decrease time to provoked reaction and therefore decrease length of challenge and desensitization protocol. ${ }^{9,12}$ Addition of this method to the protocol described here may further decrease protocol length and should be explored. 


\section{One-day aspirin challenge and desensitization protocol}

Ninety-three percent of our patients were able to achieve a desensitized state within one day. The average amount of time it took to complete the protocol was 9 hours and 29 minutes. This is significantly less than the ketorolac-aspirin protocol (1.9 days or approximately 15 hours) or the three-hour oral aspirin protocol (2.6 days or approximately 21 hours). Even in the one-hour dose escalation protocol, only $40 \%$ of patients were able to finish in one day. This is likely because though the escalation interval was shorter, the protocol included five, rather than four different dose administrations, and stacking of dose administrations may have triggered more bronchial reactions that required the patient to return the following day to continue testing. It should be noted that due to limitations in operating hours, some clinics may require more than one day to complete the protocol.

\section{Safety and tolerability}

In comparison to the standard 3-hour escalation protocol, our one-day, 90-minute oral aspirin desensitization protocol had a similar safety profile. No patients from either protocol required emergency department visit or hospitalization. The mean decline in FEV1 (18.2 vs $13.4 \%$ ) and rate of patients with severe bronchial reaction defined as decline in FEV1 $>30 \%$ (16 vs 11\%) were similar between in the 90-minute and 3-hour protocol, respectively. Among 90-minute protocol patients, there were more patients who only experienced nasoocular reaction ( 50 vs $35 \%$ ) and fewer with GI reaction (23 vs $33 \%$ ). Cutaneous reaction was more common in the 90-minute oral aspirin group (18 vs $10 \%) .{ }^{6}$ It is important to note that aspirin challenge and desensitization protocols should be administered by physicians and other medical staff who are trained in protocol implementation and management of allergies and anaphylaxis.

\section{Limitations}

Limitations of this study include that the participants for whom our current recommendations are based met the specific inclusion/exclusion criteria outlined above; patients were required to be on a stable asthma regimen with baseline FEV1 $\geq 70 \%$. This may have narrowed our sample to those patients at a lower risk for severe adverse events and it is therefore unknown if this protocol can be universally applied to all patients with AERD, regardless of asthma control. However, the protocol described here is now used widely at the Brigham and Women's Hospital AERD Center, even for some patients who do not strictly meet these criteria, including FEV1<70\%, so we are optimistic that a study inclusive of higher risk patients would yield similar results. The study population was primary Caucasian which limits generalizability of findings to non-white ethnic and racial groups.

\section{Conclusion}

Patients with AERD on a stable asthma regimen and with a baseline FEV1 $\geq 70 \%$ can be safely desensitized to aspirin using a 90-minute dose escalation protocol, starting at a dose of $40.5 \mathrm{mg}$, and defining desensitization as tolerance of the repeated provocation dose and at least one subsequent aspirin dose, bringing total cumulative daily dose to $2325 \mathrm{mg}$. This protocol can routinely be completed in one day. 


\section{Acknowledgments}

Funding: This work was supported by the National Institutes of Health (NIH grant nos U19AI095219,

K23HL111113, K23AI118804, R01HL128241, T32AI00730), and by generous contributions from the Vinik and

Kaye Families.

\section{ABBREVIATIONS}
(AERD)
aspirin-exacerbated respiratory disease
(NSAIDs)
non-steroidal anti-inflammatory drugs
(TNSS)
total nasal symptoms score
(FEV1)
forced expiratory volume in 1 second
(ACQ)
asthma control questionnaire

\section{REFERENCES}

1. Stevenson DD, Simon RA. Selection of patients for aspirin desensitization treatment. J Allergy Clin Immunol. 2006;118(4):801-804. [PubMed: 17030229]

2. Stevenson DD. Diagnosis, prevention, and treatment of adverse reactions to aspirin and nonsteroidal anti-inflammatory drugs. J Allergy Clin Immunol. 1984;74(4 Pt 2):617-622. [PubMed: 6436354]

3. Macy E, Bernstein JA, Castells MC, Gawchik SM, Lee TH, Settipane RA, et al. Aspirin challenge and desensitization for aspirin-exacerbated respiratory disease: a practice paper. Ann Allergy Asthma Immunol. 2007;98(2):172-174. [PubMed: 17304886]

4. Joint Task Force on Practice P, American Academy of Allergy A, Immunology, et al. Drug allergy: an updated practice parameter. Ann Allergy Asthma Immunol. 2010;105(4):259-273. [PubMed: 20934625]

5. Chen JR, Buchmiller BL, Khan DA. An Hourly Dose-Escalation Desensitization Protocol for Aspirin-Exacerbated Respiratory Disease. J Allergy Clin Immunol Pract. 2015;3(6):926-931 e921. [PubMed: 26282158]

6. Lee RU, White AA, Ding D, Dursun AB, Woessner KM, Simon RA, et al. Use of intranasal ketorolac and modified oral aspirin challenge for desensitization of aspirin-exacerbated respiratory disease. Ann Allergy Asthma Immunol. 2010;105(2):130-135. [PubMed: 20674823]

7. White A, Bigby T, Stevenson D. Intranasal ketorolac challenge for the diagnosis of aspirinexacerbated respiratory disease. Ann Allergy Asthma Immunol. 2006;97(2):190-195. [PubMed: 16937750]

8. Patriarca G, Bellioni P, Nucera E, Schiavino D, Papa G, Schinco G, et al. Intranasal treatment with lysine acetylsalicylate in patients with nasal polyposis. Ann Allergy. 1991;67(6):588-592. [PubMed: 1750721]

9. Pelletier T, Roizen G, Ren Z, Hudes G, Rosenstreich D, Jerschow E. Comparable safety of 2 aspirin desensitization protocols for aspirin exacerbated respiratory disease. J Allergy Clin Immunol Pract. 2018.

10. Hope AP, Woessner KA, Simon RA, Stevenson DD. Rational approach to aspirin dosing during oral challenges and desensitization of patients with aspirin-exacerbated respiratory disease. $\mathrm{J}$ Allergy Clin Immunol. 2009;123(2):406-410. [PubMed: 19056109]

11. Laidlaw TM, Cahill KN, Cardet JC, Murphy K, Cui J, Dioneda B, et al. A trial of type 12 purinergic (P2Y12) receptor inhibition with prasugrel identifies a potentially distinct endotype of patients with aspirin-exacerbated respiratory disease. J Allergy Clin Immunol. 2018.

12. Feldman M, Cryer B. Aspirin absorption rates and platelet inhibition times with $325-\mathrm{mg}$ buffered aspirin tablets (chewed or swallowed intact) and with buffered aspirin solution. Am J Cardiol. 1999;84(4):404-409. [PubMed: 10468077] 
13. Cormican LJ, Farooque S, Altmann DR, Lee TH. Improvements in an oral aspirin challenge protocol for the diagnosis of aspirin hypersensitivity. Clin Exp Allergy. 2005;35(6):717-722. [PubMed: 15969660] 


\section{HIGHLIGHTS BOX}

1. What is already known about this topic?

Aspirin challenge and desensitization is the gold standard in diagnosis and treatment of patients with aspirin-exacerbated respiratory disease, but the protocols can be time and resource intensive and there are limited data regarding safety.

2. What does this article add to our knowledge?

An aspirin challenge and desensitization protocol beginning with a $40.5 \mathrm{mg}$ dose, using 90-minute dose escalation intervals, and implementing an updated definition of desensitization is safe and can routinely be accomplished within one day.

3. How does this study impact current management guidelines?

Updating the current aspirin challenge and desensitization guidelines to include these three key elements will allow for maintaining an exceptional safety profile while increasing efficiency of this procedure. 


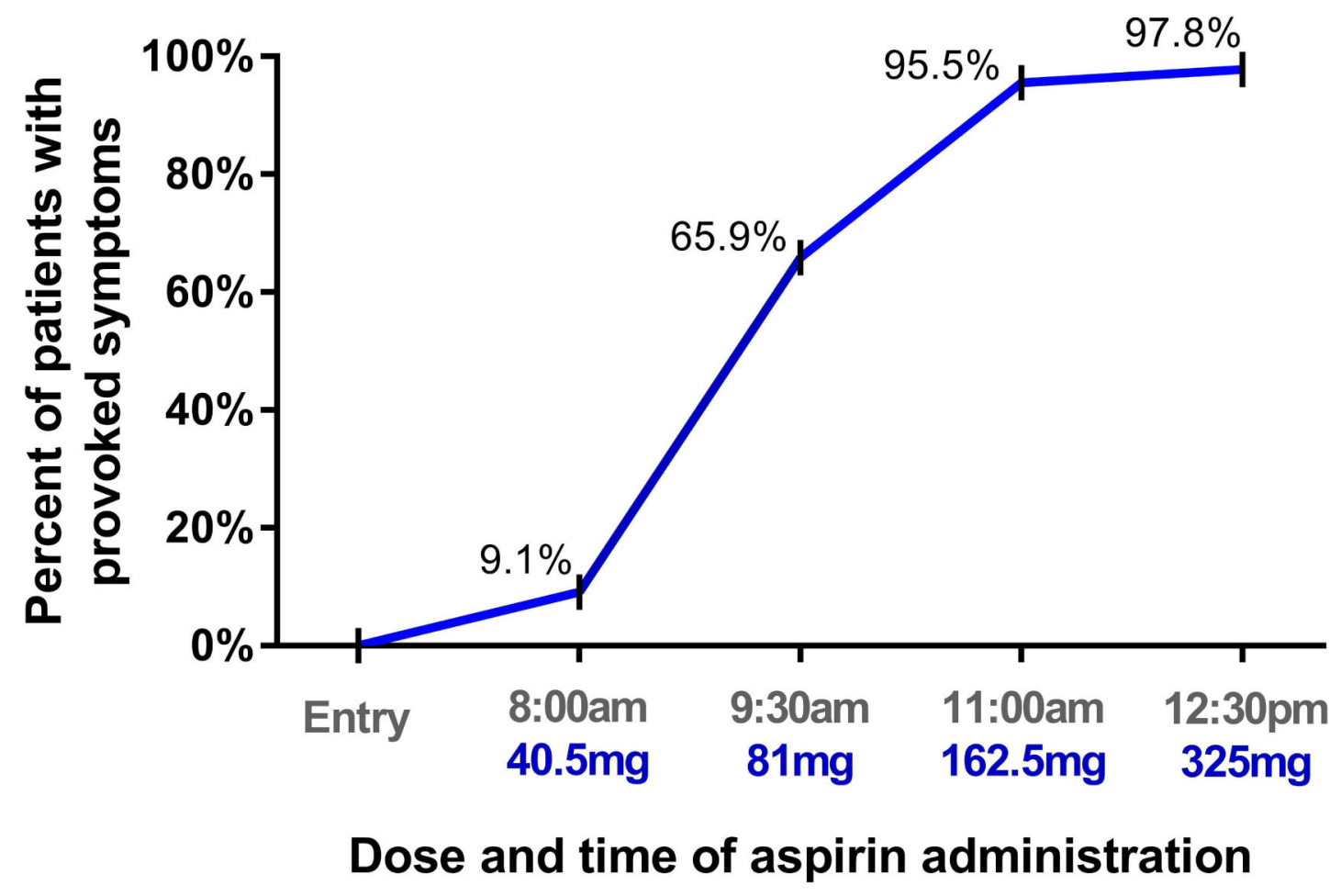

Figure 1. Rate of symptom provocation by dose.

Linear representation of the total percentage of patients whose reaction symptoms had been provoked by each aspirin dose. The final percentage is less than $100 \%$ because one of the 44 patients with previously aspirin challenge-proven AERD did not develop any symptoms of reaction at this aspirin challenge and was considered to have a "silent desensitization". 

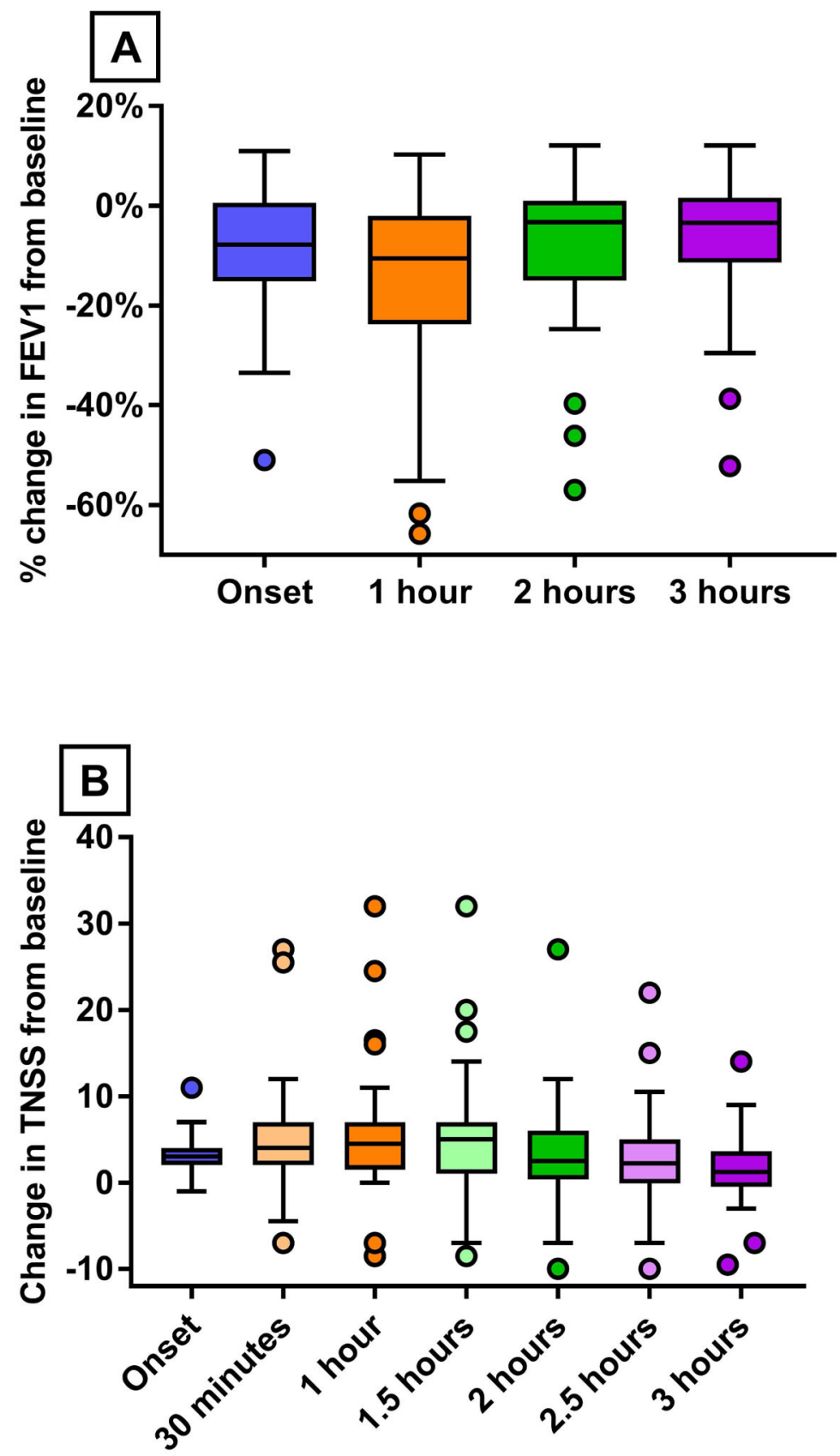

Figure 2A-B. Lower respiratory and naso-ocular reaction symptoms following provocative dose of aspirin.

A) The percent change from baseline in FEV1 at the onset of reaction symptoms and every hour during the three-hour observation period. B) The change from baseline in TNSS score at the onset of reaction symptoms and every 30 minutes during the three-hour observation period. Data are shown as box-and-whisker plots from the $25^{\text {th }}-75^{\text {th }}$ percentiles, displayed using the Tukey method. 
름
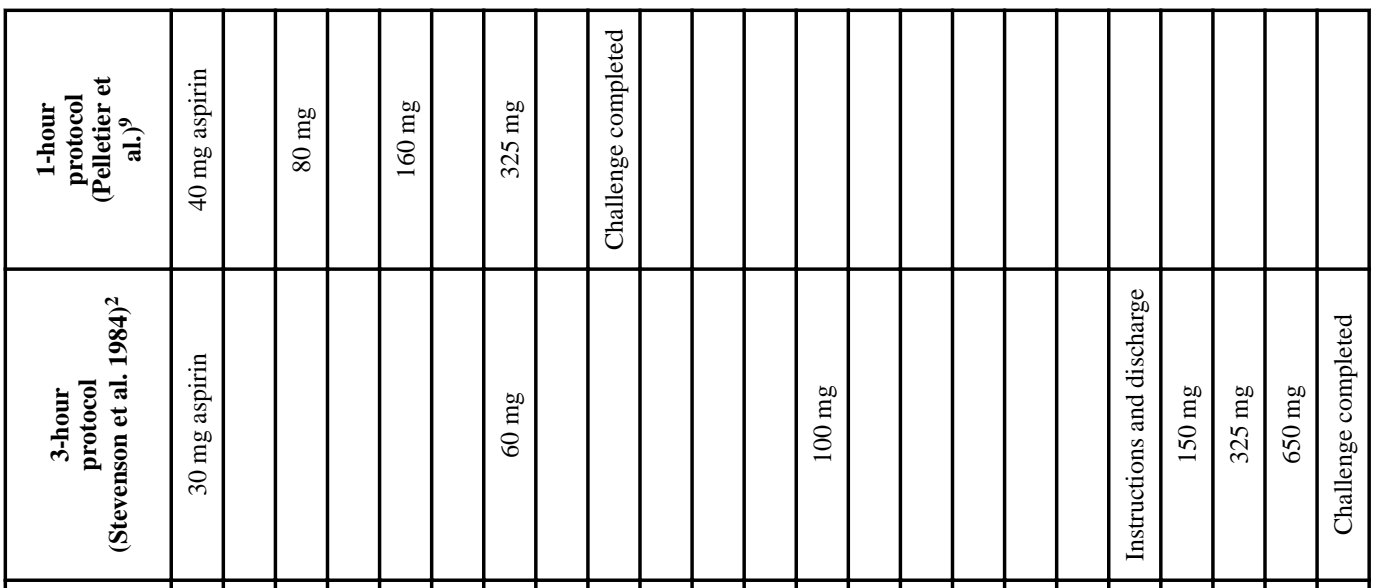

를

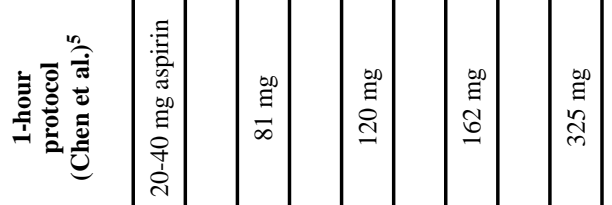

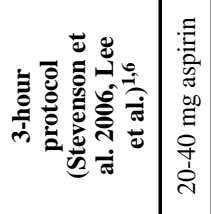

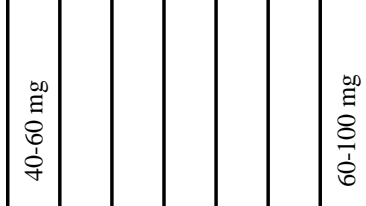

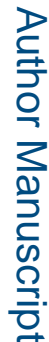
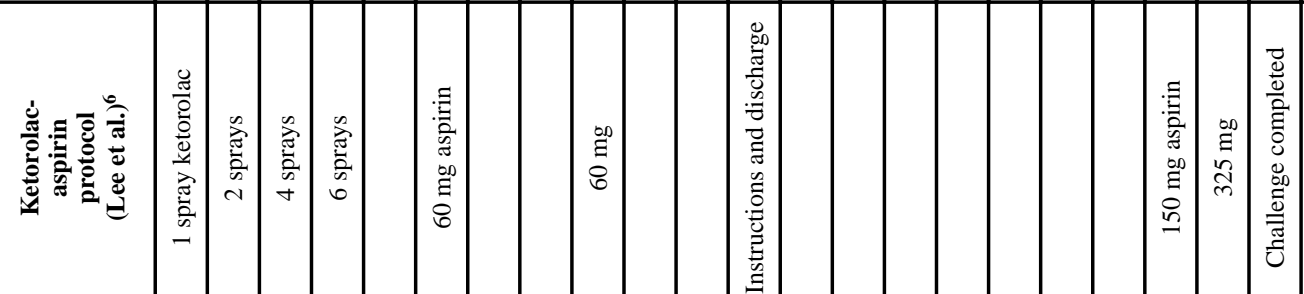

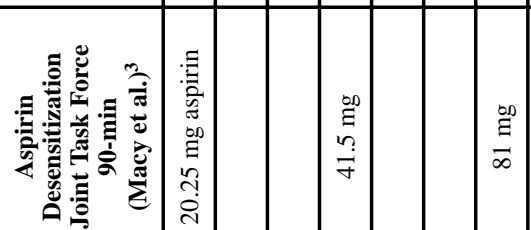

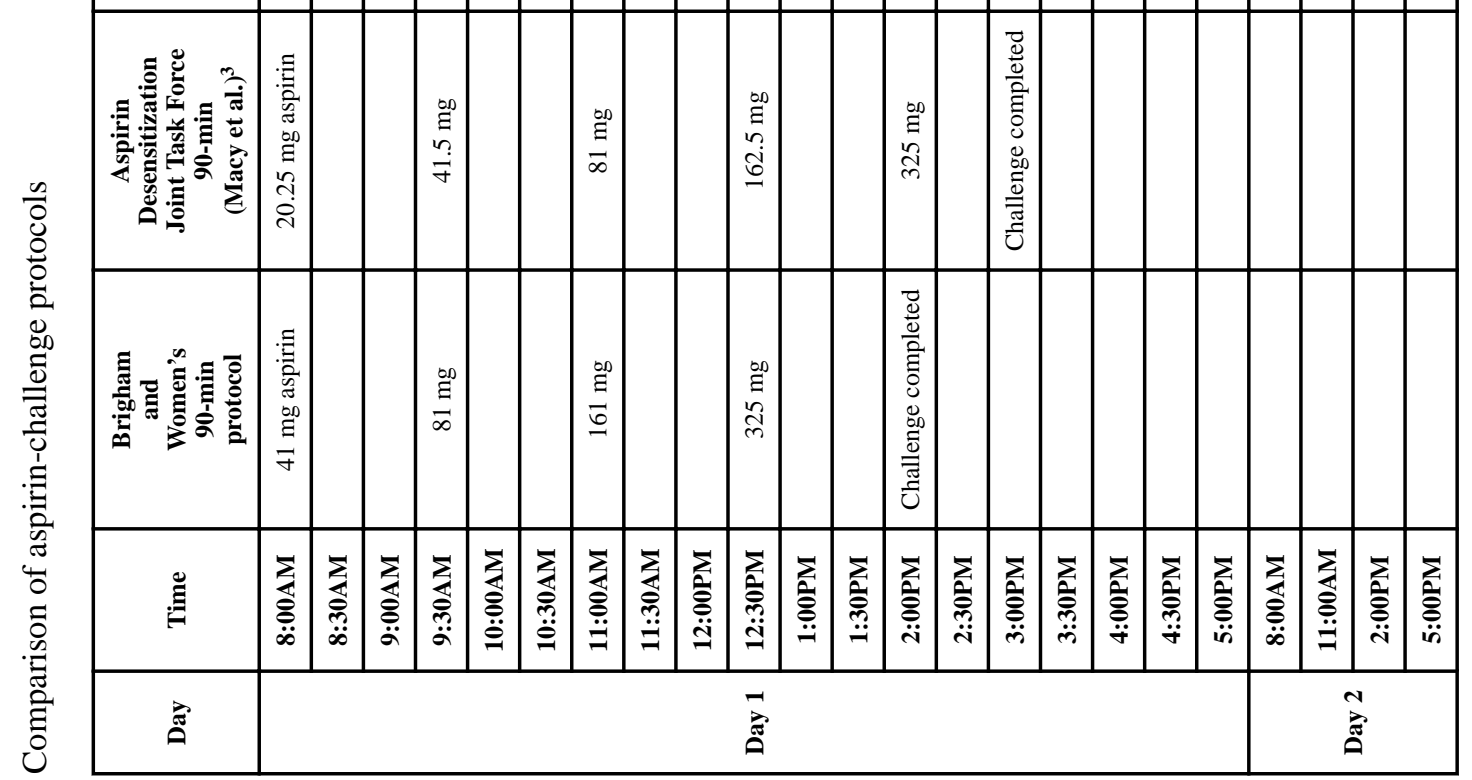

ᄅ

J Allergy Clin Immunol Pract. Author manuscript; available in PMC 2020 April 01 
Table 2.

\section{Patient Characteristics}

\begin{tabular}{lr}
\hline Characteristic & \\
\hline Number of participants & 44 \\
Age, mean (SE) & $47(1.4)$ \\
Sex, $\mathrm{n}(\%)$ & $18(41.0 \%)$ \\
$\quad$ Male & $26(59.1 \%)$ \\
$\quad$ Female & \\
Race/ethnicity, $\mathrm{n}(\%)$ & $44(100 \%)$ \\
$\quad$ Caucasian & $43(97.7 \%)$ \\
$\quad$ Non-Hispanic/Latino & $1(2.3 \%)$ \\
$\quad$ Hispanic/Latino & $91.2 \%(1.6)$ \\
Baseline percent predicted FEV1, mean (SE) & $39(88.6 \%)$ \\
Inhaled corticosteroid use, $\mathrm{n}(\%)$ & $29(67.4 \%)$ \\
Long-acting beta-agonist use, $\mathrm{n}(\%)$ & $0(0 \%)$ \\
Oral glucocorticoid, $\mathrm{n}(\%)$ & $44(100 \%)$ \\
Montelukast, $\mathrm{n}(\%)$ & $1.5(1-4)$ \\
Baseline TNSS, median $\left(25-75^{\text {th }}\right.$ percentile) & \\
Baseline ACQ, median (25-75 & \\
FEV1; percentile) & $0.43(0.29-0.86)$ \\
TNSS; Total Nasal Symptom Score & \\
ACQ; Asthma Control Questionnaire & \\
SE; Standard Error &
\end{tabular}


Table 3.

Safety, tolerability and feasibility of a one-day 90-minute dose escalation aspirin desensitization protocol

\begin{tabular}{|c|c|c|}
\hline \multicolumn{2}{|l|}{ Patient safety and tolerability } & \\
\hline Time from provocative dose to reaction, mean (SD) in minutes & 61 mins (22) & \\
\hline Reaction to repeat provoking dose, $\mathrm{n}(\%)$ & $0(0)$ & \\
\hline \multicolumn{3}{|l|}{ Upper and lower respiratory reactions } \\
\hline No reaction, $\mathrm{n}(\%)$ & $1(2.3)$ & \\
\hline Bronchial only, n (\%) & $0(0)$ & \\
\hline Naso-ocular only, n (\%) & $22(50.0)$ & \\
\hline Bronchial and naso-ocular, n (\%) & $21(47.7)$ & \\
\hline \multicolumn{3}{|l|}{ Extrapulmonary reactions } \\
\hline Gastrointestinal, n (\%) & $10(23.3)$ & \\
\hline Dermatologic, n $(\%)$ & $8(18.6)$ & \\
\hline Hypotension, n (\%) & $0(0)$ & \\
\hline \multicolumn{3}{|l|}{ Significant lower respiratory symptoms } \\
\hline No significant percent change in FEV1, $\mathrm{n}(\%)$ & $22(51.2)$ & \\
\hline Significant percent change in FEV1, n (\%) & $21(48)$. & \\
\hline \multicolumn{3}{|c|}{ Change in FEV 1 among those with significant lower respiratory symptoms } \\
\hline Minimum & $-15 \%$ & \\
\hline 25th percentile & $-18 \%$ & \\
\hline Median & $-25 \%$ & \\
\hline $75^{\text {th }}$ percentile & $-31 \%$ & \\
\hline Maximum & $-66 \%$ & \\
\hline \multicolumn{3}{|c|}{ Number of nebulizer treatments (albuterol and/oripratropium) required, $\mathrm{n}(\%)$} \\
\hline Zero nebulizer treatments & $20(45.5)$ & \\
\hline One nebulizer treatments & $8(18.6)$ & \\
\hline Two nebulizer treatments & $8(18.6)$ & \\
\hline Three or more nebulizer treatments & $7(16.3)$ & \\
\hline \multicolumn{3}{|l|}{ Feasibility of completion within 1-day } \\
\hline Reached desensitization within one day, $\mathrm{n}(\%)$ & & $41(93.2)$ \\
\hline Did not reach desensitization in one day, $\mathrm{n}(\%)$ & & $3(6.8)$ \\
\hline Physician discontinuation for safety/tolerability & & $1(2.3)$ \\
\hline Patient choice & & $2(4.6)$ \\
\hline Average length of time per challenge/desensitization completed & n 1 day (range) & 9 hours and 29 mins ( $7 \mathrm{hrs} 20 \mathrm{mins}-12 \mathrm{hrs}$ ) \\
\hline
\end{tabular}

J Allergy Clin Immunol Pract. Author manuscript; available in PMC 2020 April 01. 